FORMATION Formation emploi

Revue française de sciences sociales

118 | avril-juin 2012

10 ans de parcours professionnels des jeunes :

l'intérêt des études longitudinales

\title{
Postface : Quelles politiques contre le décrochage et le déclassement?
}

Eric Maurin

\section{(2) OpenEdition \\ Journals}

Édition électronique

URL : http://journals.openedition.org/formationemploi/3653

ISSN : 2107-0946

Éditeur

La Documentation française

Édition imprimée

Date de publication : 30 juin 2012

Pagination : 125-133

ISSN : 0759-6340

Référence électronique

Eric Maurin, « Postface : Quelles politiques contre le décrochage et le déclassement ? », Formation emploi [En ligne], 118 | avril-juin 2012, mis en ligne le 23 juillet 2012, consulté le 30 octobre 2020. URL : http://journals.openedition.org/formationemploi/3653 


\title{
Postface : Quelles politiques contre le décrochage et le déclassement?
}

\author{
Eric MAURIN \\ École d'Économie de Paris
}

Au sein de chaque génération, environ $12 \%$ des jeunes achèvent leur scolarité sans diplôme et se retrouvent exposés à des niveaux très élevés de chômage et de précarité. Selon les récentes enquêtes sur l'emploi, le taux de chômage des personnes sans diplôme est ainsi en moyenne de $41 \%$ dans les cinq années qui suivent la sortie de l'école, près de deux fois plus élevé que celui des titulaires d'un CAP (23\%) et plus de trois fois plus élevé que celui des bacheliers de la filière professionnelle (13\%), sans parler des bacheliers des filières générales et technologiques (10\%). Le fait que ce numéro de Formation Emploi consacre plusieurs articles à ce problème me semble tout à fait bienvenu, tant le besoin est grand d'en savoir davantage sur les causes réelles du décrochage scolaire et ses conséquences propres. Dans les lignes qui suivent, je me propose d'apporter quelques éclairages complémentaires sur ce thème, à partir de mes propres travaux ainsi que des recherches récentes en économie ; à cet effet, je me centrerai notamment sur la question des politiques publiques susceptibles de faire reculer les sorties sans qualification et d'améliorer le sort des jeunes au moment de leur transition vers le marché du travail. J'aborderai également la question du déclassement des diplômés, autre sujet central évoqué par ce dossier.

\section{Décrochage et pauvreté}

S'agissant du décrochage scolaire, certains constats sont aujourd'hui relativement bien établis et partagés. Par exemple, il n'est guère contesté que le décrochage et les sorties sans diplôme frappent avant tout les enfants de milieux déshérités. Cette réalité conduit nombre d'observateurs à militer pour des politiques publiques aidant plus particulièrement les familles à bas revenus, notamment au moment où leurs enfants arrivent en fin de scolarité obligatoire, à l'âge du décrochage. Le Royaume-Uni a récemment expérimenté avec succès un système d'allocations versées aux familles modestes dont les enfants poursuivent une formation après 16 ans, à l'issue de leur scolarité obligatoire (Dearden et al., 2009). La pauvreté est très souvent synonyme d'habitat dégradé et de logement surpeuplé, 
avec plusieurs enfants par chambre. Il s'agit de l'un des canaux essentiels par lequel le manque de ressources des parents hypothèque la scolarité des adolescents et favorise le désinvestissement scolaire (Goux et Maurin, 2005). Il est très difficile de se construire et de progresser quand on n'a pas d'espace à soi à la maison. Les politiques du logement devraient viser explicitement à réduire le surpeuplement des logements dont souffrent aujourd'hui plus de $20 \%$ des enfants.

Autre diagnostic partagé, le décrochage en cours d'études secondaires est très souvent le point d'aboutissement d'un processus de désengagement scolaire amorcé bien des années plus tôt, au primaire voire même en maternelle. La quasi-totalité des décrocheurs ont d'ailleurs redoublé au moins une fois avant de lâcher prise. Cet enracinement du décrochage dans les parcours scolaires amène aujourd'hui de nombreux spécialistes à privilégier des politiques menées le plus en amont possible, dès le plus jeune âge (pour une défense de ce point de vue, voir par exemple Heckman, 2008). Beaucoup estiment aujourd'hui que les problèmes du collège et du lycée ne peuvent se résoudre qu'à l'unique condition de d'abord régler la question de l'échec scolaire au primaire. Cette vision paraît un peu extrême au regard de la difficulté rencontrée partout dans le monde à mettre en place à grande échelle un soutien scolaire précoce qui soit à la fois efficace pédagogiquement et non stigmatisant pour les petits bénéficiaires. L'expérience anglaise d'aide personnalisée pour les élèves en difficulté au primaire (dont s'est inspirée récemment la France ${ }^{1}$ ) s'avère par exemple un échec : elle génère autant d'effets négatifs de stigmatisation que d'effets positifs. Elle n'a au total aucun effet perceptible (voir Keslair, Maurin et McNally, 2011).

Qu'il s'agisse de politiques de lutte contre l'échec dans le primaire ou de politiques de soutien financier aux familles dont les enfants terminent le collège, les quelques programmes aujourd'hui identifiés comme efficaces ont souvent pour inconvénient de représenter des investissements très couteux et difficiles à assumer en période de disette budgétaire (même si l'on considère que leurs bénéfices de long terme seront considérablement plus élevés que leurs coûts immédiats). Pour vraiment augmenter les chances de succès à long terme des enfants de milieux modestes, on ne peut pas se contenter de demi-mesures. Autant un programme d'école maternelle intensif, en petits groupes, à 15000 euros par an et par enfant, peut avoir d'importants effets de long terme sur ses petits bénéficiaires, autant les versions à coûts réduits à 5000 euros par an et par enfant (par exemple la maternelle française actuelle, à trente élèves par classe) s’avèrent souvent décevantes (voir par exemple Baker et al., 2008 ; Goux et Maurin, 2010). De même, pour être efficaces et vraiment

1. Depuis 2008, le temps scolaire des écoles françaises est organisé en 24 heures d'enseignement pour tous les élèves, ceux repérés par les enseignants comme rencontrant des difficultés pouvant en outre bénéficier de 2 heures d'aide personnalisée supplémentaires de la part de ces mêmes enseignants (contre 26 heures communes pour tous les élèves avant 2008). Dans l'esprit, cette nouvelle organisation est très proche du système mis en place en Angleterre en faveur des élèves repérés par leurs enseignants comme ayant des special education needs (besoins spécifiques d'enseignement). 
modifier les comportements des décrocheurs potentiels, les allocations versées aux familles doivent être du même ordre de grandeur que les revenus d'activité espérés par les jeunes en cas de décrochage, soit l'équivalent d'un demi smic (salaire minimum interprofessionnel de croissance) ; ce qui n’est pas très élevé dans l'absolu, mais néanmoins considérablement plus élevé que les bourses aujourd'hui offertes aux collégiens et lycéens français.

\section{L'allongement obligatoire des scolarités}

Une autre politique (impliquant a priori des coûts par élève moindres) serait d'augmenter l'âge minimum de fin d'études, de passer par exemple de 16 ans à 18 ans dans le cas français. Nombre de recherches récentes démontrent les effets très vertueux qu'ont pu avoir dans le passé les augmentations d'âge minimum de fin d'études, notamment lorsqu'elles se sont accompagnées d'aides financières en direction des familles modestes, les plus directement concernées (voir par exemple l'analyse de l'expérience suédoise par Meghir et Palme, 2005, ou encore Oreopoulos, 2007). La question se pose toutefois de savoir si les publics (très pauvres et déshérités) qui seraient aujourd'hui concernés par ce type de mesure tireraient autant profit de cette politique que les familles qui furent concernées dans le passé.

Si l'on suit Grenet (2011), il faudrait au minimum faire en sorte que cet allongement imposé coïncide avec un accroissement des possibilités de certifications professionnelles à l'issue des scolarités obligatoires : on tend à apprendre mieux et de façon plus durable quand il y a un examen à la clef. Par rapport à une politique de bourses conditionnelles à l'obtention de qualifications, l'allongement obligatoire des scolarités présente néanmoins le risque de ne pas se traduire par une augmentation du nombre de jeunes qualifiés, mais de se diluer en redoublements et en trajectoires scolaires, certes plus longues, mais tout aussi avortées. D'après Grenet (2011), les politiques d'allongement obligatoire des scolarités ont eu dans le passé des effets d'autant plus positifs qu’elles ont été menées dans des pays où prévalaient une logique de certification (comme le Royaume-Uni) plutôt que dans des pays dominés par la logique du concours et du redoublement (comme la France).

En mettant en lumière les effets positifs des politiques d'allongement des scolarités obligatoires, les recherches récentes ont eu pour intérêt de battre en brèche (sur des bases objectives et pas simplement idéologiques) l'idée selon laquelle l'offre et la demande d'éducation s'équilibrent nécessairement de façon optimale pour la société, sans qu'il soit besoin de contraindre qui que ce soit. De fait, on pourrait imaginer que chacun sache parfaitement déterminer ce qui lui convient au mieux en termes d'éducation, sans avoir besoin d'être contraint par l'État à aller plus loin (conformément aux formulations initiales de la théorie du capital humain par exemple). Dans cette hypothèse, il n’y aurait aucun bénéfice particulier à attendre d'une contrainte de scolarité minimale imposée aux individus par la collectivité, au contraire. Si la contrainte s'avère finalement payante à long 
terme (y compris pour ceux que l'on force temporairement à faire ce qu'ils n'auraient pas fait spontanément), c'est que la décision initiale du décrochage scolaire n'a rien d'optimal, ni pour la société, ni pour l'adulte en devenir. Quand ils abandonnent leur scolarité, les décrocheurs ont bien souvent une perception tout à fait tronquée des possibilités de formation qui s'offrent à eux (et de leurs bénéfices potentiels), ainsi qu'une vision biaisée de l'avenir, dans laquelle les coûts actuels de poursuite des études (l'ennui, l'absence de motivations) pèsent d'un poids disproportionné par rapport aux bénéfices ultérieurs, tout au long de leur vie d'adulte, de l'obtention d'une qualification solide.

Sur ces points, d'autres expériences naturelles ${ }^{2}$ délivrent des enseignements voisins et tout aussi éloquents, comme la suppression du service militaire à la fin des années 1990 par exemple. Dans un passé pas si lointain, poursuivre ses études après 18 ans avait pour intérêt immédiat pour les garçons de permettre de reporter le moment du service militaire. En supprimant ce bénéfice immédiat, on a constaté que l'abolition du service militaire, en 1997, a entraîné un surcroît immédiat de décrochage scolaire entre 17 et 19 ans chez les garçons et par la suite une baisse sensible de leurs salaires à l'entrée sur le marché du travail, comparés à ceux des jeunes filles des mêmes générations, notamment dans les milieux modestes (Maurin et Xénogiani, 2007). Les jeunes décrocheurs ne sont pas les investisseurs hyper-rationnels postulés par la théorie du capital humain, mais bien au contraire des êtres encore en devenir, complètement myopes socialement, sous-évaluant les coûts futurs du désinvestissement scolaire et surévaluant ses bénéfices immédiats.

\section{Améliorer les processus d'orientation}

De fait, de très nombreux adolescents décrochent et se retrouvent aujourd'hui sans diplôme sur le marché du travail pour la simple raison qu'ils méconnaissent (ou perçoivent comme sans valeur) des filières professionnelles aujourd'hui délaissées alors pourtant qu'elles permettent d'accéder à des qualifications très solides. Dans ce contexte, il serait important de réhabiliter les politiques susceptibles d'améliorer la qualité des orientations, de réduire les déceptions et les rancœurs que les orientations subies (et mal comprises) nourrissent aujourd'hui chez nombre d'adolescents et dans leurs familles ( $c f$. article de Yaël Brinbaum et Christine Guegnard dans ce numéro). Une expérience en cours dans l'académie de Versailles suggère que le nombre de décrocheurs précoces (près de $10 \%$ des jeunes dans cette académie) peut être quasi divisé par deux dès lors qu'un programme relativement

2. Le terme d' "expérience naturelle » désigne les situations où les aléas de l'histoire (ou du cours naturel des choses, le sexe du premier enfant par exemple) produisent l'équivalent d'une expérience aléatoire contrôlée en laboratoire, c'est-à-dire avec groupes tests et témoins définis de façon quasi aléatoire. La suppression $\mathrm{du}$ service militaire s'apparente par exemple à une expérience de laboratoire où les jeunes nés juste après la suppression forment un groupe test et ceux nés juste avant un groupe témoin. 
léger d'information et de bilans individualisés est spécifiquement mis en place pour les $25 \%$ de collégiens les plus en difficulté en fin de quatrième. Les politiques en faveur d'orientations moins subies et mieux comprises seraient non seulement considérablement moins coûteuses que des politiques de bourses à l'anglaise ou qu'un allongement obligatoire des scolarités, mais elles seraient également tout aussi efficaces.

Il me parait d'autant plus souhaitable de travailler dans cette direction que - comme le suggèrent Jean-Paul Gehin et Ugo Palheta dans ce numéro - chaque année gagnée sur le décrochage compte. En particulier, même s'ils finissent par échouer au diplôme, les jeunes ayant fait l'effort de tenter une formation s'en tirent mieux que ceux qui ont abandonné avant même de vraiment essayer. Contrairement à une conviction très enracinée dans l'imaginaire français, la formation initiale ne produit pas qu'un classement et des "signaux ", mais accroît également notre capacité collective à progresser et créer des emplois nouveaux (Chevalier et al., 2004). Quand on analyse précisément les destinées des cohortes arrivées sur le marché du travail avant, pendant et après la dernière grande vague de démocratisation scolaire (soit les cohortes nées entre le début des années 1960 et la fin des années 1970), on constate que l'accélération puis le ralentissement brutal de la diffusion des qualifications dans ces cohortes ont coïncidé avec une amélioration puis un coup de frein tout aussi nets dans la qualité globale de leur insertion collective sur le marché du travail, phénomène impossible à comprendre si l'éducation n'était qu'un signal classant les individus au sein de chaque cohorte (voir Maurin, 2007, chapitre 5).

En résumé, chaque accroissement de 10 points de la proportion de diplômés supérieurs dans une cohorte s'accompagne d'une baisse globale d'environ 3 points du taux de chômage d'insertion et d'une hausse de 5 points du taux d'emplois très qualifiés (niveau cadre). Les organisations se renouvellent en profondeur en permanence et leur capacité à créer ou recréer des emplois qualifiés est chaque année directement indexée sur les qualifications dont sont porteurs les nouveaux arrivants sur le marché du travail. Il n'est pas besoin de croire à l'investisseur hyper avisé de la théorie du capital humain pour chercher dans les qualifications produites par le système éducatif autre chose qu'un simple signal. Sans la deuxième vague de démocratisation scolaire, à la fin des années 1980, la situation des jeunes sur le marché du travail serait aujourd'hui plus problématique qu'elle ne l'est.

\section{Peur du déclassement et déclassement stratégique}

Si l'on définit le déclassement comme une chute en bas de l'échelle sociale, l'échec scolaire représente sans doute aujourd'hui l'une des formes de déclassement les plus difficiles à surmonter, tant le diplôme est devenu nécessaire pour accéder à un quelconque statut dans notre société. Le déclassement peut toutefois survenir à d'autres moments de la vie et revêtir d'autres modalités, notamment pour tous ceux qui n'arrivent pas à s'insérer 
dans la société et obtenir un emploi stable en dépit d'études longues. Certains diplômés évitent ces formes de relégation en passant un concours de la fonction publique, se mettant définitivement à l'abri de la précarité, fut-ce au prix d'un début de carrière dans des emplois relativement sous-qualifiés. Ils consentent un déclassement que l'on peut qualifier de "stratégique " pour conjurer le spectre d'un déclassement plus radical, synonyme de pauvreté et d'exclusion.

Les déclassements stratégiques sont évidemment plus ou moins temporaires selon le type de concours réussi et de fonction publique intégrée. L'analyse de Vanessa di Paola et Stéphanie Moullet, dans ce numéro, démontre que la fréquence et la persistance du déclassement dans la fonction publique sont assez proches pour les hommes et pour les femmes. Ce constat n'allait pas de soi et enrichit notre connaissance des carrières du salariat du public. Il demeure néanmoins difficile à interpréter, tant le choix de la fonction publique obéit à des logiques différentes pour les hommes et les femmes (ce que trahit par exemple le fait que les jeunes femmes diplômées du supérieur se tournent en nombre bien plus important vers la fonction publique que leurs homologues masculins). Les populations de jeunes fonctionnaires masculins et féminins ont été filtrées par des ambitions et des craintes souvent très différentes; de sorte qu'il est bien difficile de savoir à quoi aujourd'hui attribuer les différences et les similitudes observées dans leurs carrières de fonctionnaires. En particulier, le rôle propre joué par la gestion des carrières masculines et féminines au sein des différents organismes publics me parait difficile à identifier sur la base d'analyses qui ne corrigeraient pas la sélection différentielle dont sont issues les populations féminines et masculines travaillant dans le public.

Commele suggèrent fort justement Thomas Couppié, Arnaud Dupray et Stéphanie Moullet à la fin de leur contribution, on ne peut pas vraiment expliquer la situation relative des hommes et des femmes au sein des différents métiers sans d'abord identifier les « éventuels comportements d'auto-sélection » qui ont infléchi leurs choix de formation puis leurs choix professionnels.

De façon plus générale, on peut difficilement comprendre l'emprise du déclassement sur une société sans cerner d'abord la façon dont la peur du déclassement s'y décline, façonne les imaginaires et, partant, les choix scolaires, professionnels ou même résidentiels et matrimoniaux. Des phénomènes aussi structurants pour la dynamique d'une société que les déclassements stratégiques consentis par la jeunesse diplômée (ou la recherche d'entre-soi résidentiel et scolaire qui obsède les classes sociales supérieures) ne me semblent pas être compréhensibles sans prendre en compte la façon dont la peur du déclassement traverse cette société, notamment durant les périodes de récession et de crise, telles que celles que nous traversons en ce moment.

Dans ces périodes, le décrochage scolaire ou la perte d'emploi représentent soudain des formes d'échec beaucoup plus longues et difficiles à surmonter qu'à l'habitude, la peur de les subir devient plus forte et universelle encore, instillant dans l'ensemble de la société un éventail de stratégies de repli qui en marquent ensuite durablement le climat et les 
contours. Lors de la récession de 1993, on a pu par exemple constater une inflexion très nette des comportements des jeunes diplômés, se détournant du secteur privé pour se porter en masse vers le secteur public, modifiant en profondeur la sociologie de ce secteur (Maurin, 2009). La lutte contre la peur du déclassement (et contre les replis stratégiques qu'elle motive) passe par des politiques qui atténueraient l'irréversibilité des transitions et des échecs à l'école et sur le marché du travail ${ }^{3}$ : rien n'est plus difficile à promouvoir dans une société comme la nôtre, tout entière organisée autour de logiques de statut.

\section{Quelques questions de méthode}

Dans l'ensemble des contributions à ce numéro, l'article de Olivier Joseph, Ariane Pailhé, Isabelle Recotillet et Anne Solaz essaie de tirer parti d'une réforme récente (celle du dispositif d'allocation parentale) pour éclairer une question de fond, à savoir l'impact causal des interruptions d'activité sur les carrières salariales féminines. Il me semble qu'il s'agit d'une excellente initiative. L'évaluation des politiques publiques a mauvaise presse chez nombre de chercheurs en sciences sociales qui - entre autres griefs - ne voient guère l'intérêt pour leur discipline d'identifier les effets de telle ou telle initiative politique particulière, toujours un peu trop située et datée. Il s'agit, je crois, d'une vision tronquée, méconnaissant le fait que les initiatives de politiques publiques sont autant de quasi-expériences en vraie grandeur, permettant d'éclairer de façon souvent très crédible les relations de cause à effet existant entre les comportements (collectifs ou individuels) et les contextes dans lesquels ils prennent place. Chercher à évaluer la dernière grande période de démocratisation du système éducatif français, ce n'est pas seulement se donner les moyens de juger une politique particulière (datée et située), c’est aussi apporter des éléments inédits au vieux débat fondamental sur la nature de ce que produit le système éducatif, capital humain ou simple signal. Plus faciles à mobiliser, les modèles « toutes choses égales par ailleurs " ont la préférence des chercheurs alors pourtant que les conditions sous lesquelles ils identifient des effets causaux sont souvent très peu crédibles. En réalité, plus on raisonne " toutes choses égales par ailleurs ", plus on s'expose à interpréter comme effets causaux de purs effets de sélection.

Un dernier mot sur les données : l'unité des contributions à ce dossier tient aux enquêtes Générations qu'elles utilisent toutes. Comme le savent bien les auteurs, la principale limite de ce type de panel tient à l'attrition potentiellement très sélective qui fait fondre

3. De telles politiques pourraient sans doute s'inspirer de certaines de celles menées dans les pays scandinaves. Au Danemark, par exemple, chacun a la possibilité de retourner se former tout au long de sa vie tout en bénéficiant d'un véritable salaire de formation sur des périodes pouvant atteindre six ans. Ce type de dispositif désamorce l'enjeu d'un départ initial précoce du système éducatif et contribue sans doute à faire du Danemark l'un des pays où la peur de l'avenir est la moins répandue. 
le nombre de répondants au fil des vagues d'enquêtes. Cette attrition étant susceptible d'être liée aux phénomènes étudiés (choix de scolarité, décision de carrière), il en découle une incertitude sur la représentativité des échantillons de survivants et la portée des diagnostics statistiques ${ }^{4}$. Je trouverais intéressant que les ordres de grandeur obtenus avec cette source soient systématiquement confrontés avec ceux obtenus à partir d'enquêtes qui, comme l'enquête Emploi (ou l'enquête FQP - formation et qualification professionnelles), ne souffrent pas d'attrition, tout en permettant elles aussi une mesure assez fine des scolarités, de l'expérience professionnelle ou des situations d'emploi. En parallèle, je trouverais également pertinent de se centrer davantage encore sur ce qui fait le point fort des enquête Génération, à savoir leur dimension proprement longitudinale et l'éclairage unique qu'elles permettent de jeter sur la façon souvent chaotique dont se construisent aujourd'hui, dans le temps, les carrières.

\section{Bibliographie}

Baker M., Gruber J. and Milligan K. (2008), "Universal Childcare, Maternal Labor and Family Well-Being", Journal of Political Economy, 116 (4).

Chevalier A., Colm H., Walker I. et Zhu Y. (2004), "Does education raise productivity or just reflect it?", Economic Journal, vol. 114.

Dearden L., Emmerson C., Frayne C. and Meghir C. (2009), "Conditional Cash Transfers and School Dropout Rates", Journal of Human Resources, 44(4).

Goux D. et Maurin E. (2010), "Public School Availability for Two-year Olds and Mothers' Labour Supply”, Labour Economics, vol. 17 (6).

Goux D. et Maurin E. (2005), "The Effect of Overcrowded Housing on Children's Performance at School", Journal of Public Economics, vol. 89 (5-6).

Heckman J. (2008), "School, Skills and Synapses", Economic Inquiry, 46 (3).

GRENET J. (2011), "Is it enough to increase compulsory education to raise earnings? Evidence from French and British Compulsory Schooling Laws" (à paraittre in Scandinavian Journal of Economics).

Keslair F., Maurin E. et McNally S. (2011), "Every child matters ? An evaluation of special education needs program in England", IZA DP 6069.

Maurin E. (2009), La peur du déclassement, Seuil, Paris.

4. Dans la mesure où l'attrition frappe surtout les moins diplômés et dans l'hypothèse où elle concerne aussi en général plutôt ceux dont l'insertion se passe le moins bien, on peut par exemple craindre que l'enquête conduise à sous-estimer le rôle des diplômes et l'impact de l'éducation sur la qualité des insertions. Il est par définition très difficile de mesurer et, le cas échéant, de corriger cette forme de biais. 
Maurin E. (2007), La nouvelle question scolaire, Seuil, Paris.

Maurin E. et Xénogiani T. (2007), "Demand for Education and Labour Market Outcomes: Lessons from the Abolition of Compulsory Conscription in France", Journal of Human Resources, vol. 42 (4).

Meghir C. and Palme M. (2005), "Educational reform, Ability and Parental Background", American Economic Review, 95 (1).

Oreopoulos P. (2007), "Do dropouts dropout too soon? Wealth, Health and Happiness from Compulsory Schooling”, Journal of Public Economics, 91(11-12). 